\title{
News on Leptogenesis ${ }^{1}$
}

\author{
P. Di Bari \\ IFAE, Universitat Autònoma de Barcelona, 08193 Bellaterra (Barcelona), Spain \\ e-mail:dibari@ifae.es
}

\begin{abstract}
The possibility to explain the CMB measurement of the baryon asymmetry with leptogenesis results in a stringent bound on neutrino masses such that $\sqrt{m_{1}^{2}+m_{2}^{2}+m_{3}^{2}}<0.30 \mathrm{eV}$. We discuss the implications of such a bound for future experiments on the absolute neutrino mass scale.
\end{abstract}

\section{THE BARYON ASYMMETRY OF THE UNIVERSE}

The observation of the acoustic peaks in the power spectrum of CMB temperature anysotropies confirms that we live in a baryon asymmetric Universe, an important result already inferred from the study of cosmic rays and primordial nuclear abundances. Within standard BBN (SBBN) any measurement of a primordial nuclear abundance leads to a measurement of the baryon asymmetry, conveniently expressed in the form of baryon to photon number ratio. From the measurement of the Deuterium primordial abundance in Quasar absorption systems one finds at $1 \sigma[3]:$

$$
\left.\eta_{B 0}^{S B B N}\right|_{D / H}=(5.6 \pm 0.5) \times 10^{-10}
$$

A multiple measurement of different primordial abundances represents a test of consistency for SBBN and in principle should lead to a more accurate determination of $\eta_{B 0}$. However the results from the Helium and Litium primordial abundances are only marginally consistent with the Deuterium abundance and thus it is necessary to account for larger sistematic uncertainties and to make some assumptions on their statistical distribution. Thus an acceptable agreement among the abundances leads to a less precise determination of the baryon asymmetry [4]

$$
\eta_{B 0}^{S B B N}=(2.6-6.2) \times 10^{-10}
$$

valid approximately at the $90 \%$ c.1. [5]. The difficulty of SBBN in explaining simultaneously all the current measurements of primordial abundances can also be interpreted as a hint for the presence of non standard BBN effects. Some of them are well motivated within those models

\footnotetext{
${ }^{1}$ Proceedings of the Third Tropical Workshop, Neutrinos, Branes and Cosmology, San Juan, Puerto Rico, 19-23 August, 2002. Based on the reference papers [1],[2].
} 
beyond the Standard Model that can incorporate the see-saw and lead to leptogenesis. In any case a determination of the baryon asymmetry from SBBN, at a higher level of accuracy than the range (2), encounters serious obstacles at the present.

Fortunately the recent observation of acoustic peaks in the power spectrum of CMB temperature anysotropies provides a powerful tool to measure the baryon asymmetry and to circumvent the difficulties of SBBN. In this case one has a good consistency of different determinations of the baryon asymmetry from 6 different experiments employing different tecniques [6, 7]. A recent combined analysis gives [7]

$$
\eta_{B 0}^{C M B}=\left(6.0_{-1.1}^{+0.8}\right) \times 10^{-10}
$$

This determination is in reasonable agreement with that one from the SBBN and at the same level of accuracy. However, in contrast with the SBBN determination, the consistency of the different experimental results so far makes it quite robust and makes possible to expect a reduction of the error in a close future: below the 10\% level from the MAP satellite during next years and at the $1 \%$ level from the Planck satellite before the end of this decade. We will therefore use the CMB determination of the baryon asymmetry in our following considerations.

\section{BASICS OF LEPTOGENESIS}

Leptogenesis [8] is the cosmological consequence of the see-saw mechanism. This explains the lightness of neutrino masses by the existence of three RH neutrinos, $N_{i}$, much heavier than the electroweak scale. The decay of the heavy neutrinos violates lepton number and, in general, also CP conservation, while the cosmological expansion can yield the necessary departure from thermal equilibrium: all three Sacharov's conditions are satisfied and a lepton number can be generated in the early Universe. The possibility for leptogenesis to explain the observed baryon asymmetry relies crucially on the existence of the non perturbative SM sphaleron processes, that can convert, at temperatures above the electroweak phase transition, about $-1 / 3$ of the lepton number into a baryon number, while keeping B-L constant. The source of CP violation is naturally provided by the complexity of the neutrino mass matrices in the see-saw. For each of the three $N_{i}$ one can introduce a CP asymmetry parameter defined as:

$$
\varepsilon_{i} \equiv \frac{\Gamma_{i}-\bar{\Gamma}_{i}}{\Gamma_{i}+\bar{\Gamma}_{i}},
$$

where $\Gamma_{i}$ and $\bar{\Gamma}_{i}$ are the decay rates of $N_{i}$ respectively into leptons $\left(N_{i} \rightarrow l+\bar{\phi}\right)$ and anti-leptons $\left(N_{i} \rightarrow \bar{l}+\phi\right)$.

The problem is greatly simplified if one assumes that only the decays of the lightest RH neutrinos, $N_{1}$, can influence the final baryon asymmetry. This is true if the asymmetries generated 
by the two heavier neutrino decays (with masses $M_{2}$ and $M_{3}$ ), even though not negligible, are subsequently washed out by the processes (for example inverse decays) in which the lightest right-handed neutrinos (with mass $M_{1}$ ) are involved, at temperatures $T \sim M_{1}$. This assumption implies the existence of a mild hierarchy of masses such that $M_{2,3} \gtrsim(2-3) M_{1}$ and also that the wash out $N_{1}$-processes are strong enough.

In this way one has to solve a system of only two Boltzmann equations, one for the number of $N_{1}$ 's and one for the $B-L$ asymmetry. Introducing the convenient variable $z \equiv M_{1} / T$, they can be written in the following simple form $[9,10,1]$ :

$$
\begin{aligned}
\frac{d N_{N_{1}}}{d z} & =-(D+S)\left(N_{N_{1}}-N_{N_{1}}^{\mathrm{eq}}\right) \\
\frac{d N_{B-L}}{d z} & =-\varepsilon_{1} D\left(N_{N_{1}}-N_{N_{1}}^{\mathrm{eq}}\right)-W N_{B-L} .
\end{aligned}
$$

There are four classes of processes that contribute to the different terms in the equations: decays, inverse decays, $\Delta L=1$ scatterings and $\mathrm{RH}$ neutrino mediated processes. The first three contribute all together to modify the $N_{1}$ abundance. Indicating with $H$ the expansion rate, the term $D \equiv \Gamma_{D} /(H z)$ accounts for the decays and inverse decays while the term $S \equiv \Gamma_{S} /(\mathrm{Hz})$ accounts for the $\Delta L=1$ scatterings. The decays are also the source term for the generation of the $B-L$ asymmetry, the first term in the second equation, while all the other processes contribute to the wash out term $W \equiv \Gamma_{W} /(H z)$ that competes with the decay source term.

\section{A MODEL INDEPENDENT PARAMETERIZATION}

From the Eq. (6) it is easy to see that the solution $N_{B-L}(z)$ has to depend linearly on $\varepsilon_{1}$, in a way that the final baryon asymmetry can be written in the form

$$
N_{B-L}^{\mathrm{fin}}=N_{B-L}^{\mathrm{in}}-\frac{3}{4} \varepsilon_{1} \kappa_{0}
$$

Assuming that the wash out processes are strong enough to erase an initial value of $N_{B-L}$, generated for example by the decays of the two heavier RH neutrinos or by some other unspecified mechanism, we will put $N_{B-L}^{\text {in }}=0$. This assumption is valid under the same conditions for which heavier neutrino decays can be neglected and therefore it does not introduce further restrictions.

The efficiency factor $\kappa_{0}$ does not depend on $\varepsilon_{1}$. It is normalized in a way to be 1 in the limit case that an initial thermal abundance of $N_{1}$ 's decays fully out of equilbrium at the time when all wash out processes are completely frozen. In this limit the wash out term in the kinetic equations is uneffective and can be neglected. Let us introduce the quantity:

$$
\bar{m}=\sqrt{m_{1}^{2}+m_{2}^{2}+m_{3}^{2}}
$$


The quadratic mean of the light neutrino masses is simply $\bar{m} / \sqrt{3}$. A remarkable fact is that for masses $\left.M_{1} \ll 10^{14} \mathrm{GeV}(0.1 \mathrm{eV}) / \bar{m}\right)^{2}$ the three terms $D, S$ and $W$ are proportional to an effective neutrino mass $\widetilde{m}_{1}$ times a function of $z$ alone [10]. This means that the final baryon asymmetry, for small $M_{1}$, will depend only on two parameters: $\varepsilon_{1}$ and $\widetilde{m}_{1}$. In this case the out of equilibrium limit is obtained for $\widetilde{m}_{1} \rightarrow 0$. In figure 1 we show the function $\kappa_{0}$ as a function of $\widetilde{m}_{1}$, for different values of $M_{1}$. It can be seen how for small values of $M_{1}$ there is no dependence on $M_{1}$ itself. We performed the calculations both for an initial thermal abundance (thin lines) and for a zero initial abundance (thick lines). It is evident how there is a critical value of $\widetilde{m}_{1}$ that separates two different regimes. For $\widetilde{m}_{1} \ll 5 \times 10^{-4} \mathrm{eV}$ one recovers the limit of out of equilibrium decays and $\kappa_{0}$ is strongly dependent on the number of initial $N_{1}$ 's. In the case of zero initial neutrinos, $\kappa_{0}$ is determined by the number of $N_{1}$ 's that are produced by inverse decays and scatterings and this number goes to zero in the limit $\widetilde{m}_{1} \rightarrow 0$. Therefore in this regime there is a strong dependence on the initial conditions. For $\widetilde{m}_{1} \gg 5 \times 10^{-4} \mathrm{eV}$ there is no dependence on the initial conditions and, even for a zero initial number of $N_{1}$ 's, they are rapidly produced and their number rapidly approaches the thermal value. This means that, in the limit of large values of $\widetilde{m}_{1}$, the dependence of $\kappa_{0}$ on the $N_{1}$ production processes disappears and only a dependence on the wash out processes is left.

In the intermediate regime the value of $\kappa_{0}$ is determined by an interplay between the wash out processes and the number of decaying $N_{1}^{\prime} s$, determined both by the initial number and by the strenght of production processes. It is possible to give a numerical fit of $\kappa_{0}$ :

$$
\kappa_{0}=f_{-}\left(x_{-}\right) e^{-x_{-}}+f_{+}\left(x_{+}\right) e^{-x_{+}}
$$

with $x_{ \pm}=\left(\widetilde{m}_{1} / \widetilde{m}_{ \pm}\right)^{\alpha_{ \pm}}$. The first term depends on the initial $N_{1}$ abundance: for an initial zero abundance

$$
f_{-}\left(x_{-}\right)=f_{+}\left(x_{-}\right)=0.24 x_{-}, \widetilde{m}_{-}=3.5 \times 10^{-4} \mathrm{eV}, \alpha_{-}=0.9 ;
$$

for an initial thermal abundance

$$
f_{-}\left(x_{-}\right)=1, \widetilde{m}_{-}=4.0 \times 10^{-4} \mathrm{eV}, \alpha_{-}=0.7 .
$$

The second term is independent on the initial conditions:

$$
f_{+}\left(x_{+}\right)=0.24 x_{+}, \quad \widetilde{m}_{+}=8.3 \times 10^{-4} \mathrm{eV}, \quad \alpha_{+}=-1.1 .
$$

In the limit of weak coupling $\left(\widetilde{m}_{1} \ll 5 \times 10^{-4} \mathrm{eV}\right)$ one has $\kappa_{0} \simeq f_{-}\left(x_{-}\right)$, while in the limit of strong coupling $\left(\widetilde{m}_{1} \gg 5 \times 10^{-4} \mathrm{eV}\right)$ one has:

$$
\kappa_{0} \simeq f_{+}\left(x_{+}\right) \simeq 10^{-4}\left(\frac{\mathrm{eV}}{\widetilde{m}_{1}}\right)^{1.1} .
$$

The two fits are optimal for $M_{1}=10^{8} \mathrm{GeV}$ and are represented in figure 1 with circled lines. It is interesting to compare these results with an analytical approximation for $\kappa_{0}$ originally derived 


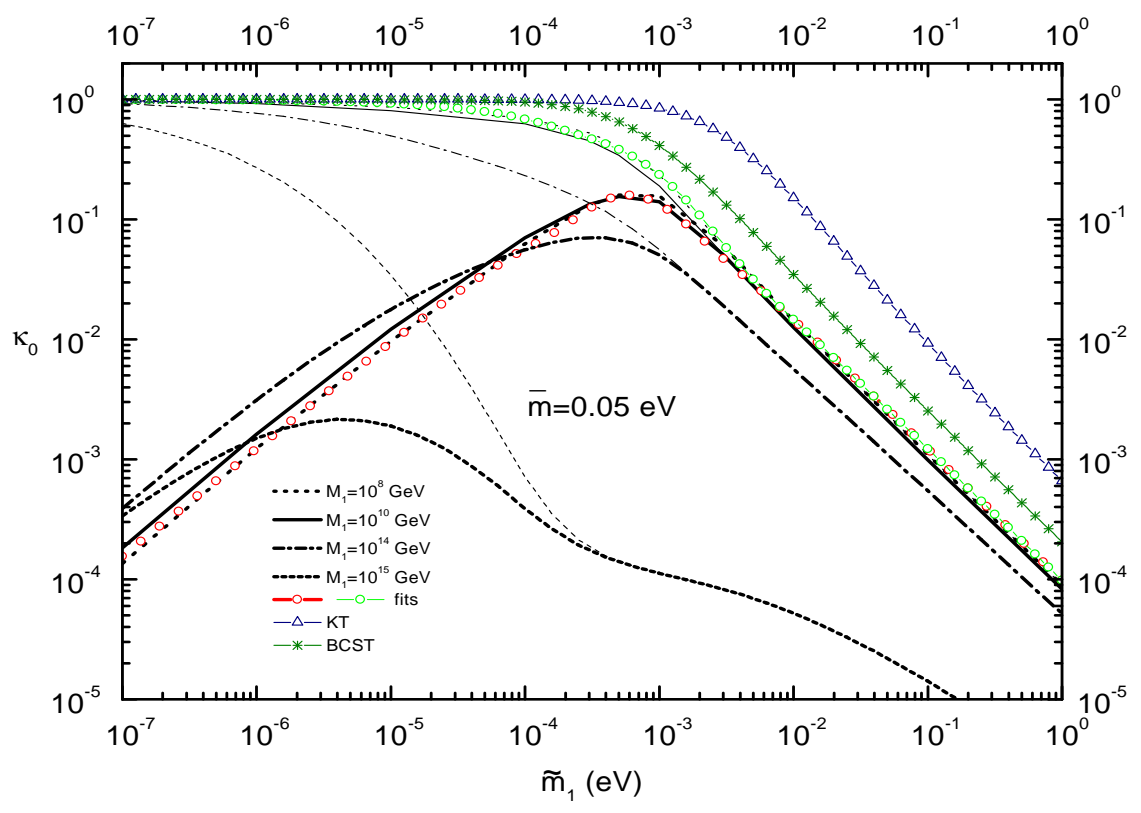

FIGURE 1. The efficiency factor.

in the context of GUT baryogenesis [11] but also adapted to the case of leptogenesis (see for example [12]):

$$
\begin{aligned}
& \kappa_{0}=1 \text { for } K \ll 1 \\
& \kappa_{0}=\frac{0.3}{K(\ln K)^{0.6}} \text { for } K \gg 1
\end{aligned}
$$

As a simple interpolating expression we can use:

$$
\kappa_{0}=\frac{0.3}{K[\ln (1+K)]^{0.6}}\left(1+\frac{0.3}{K[\ln (1+K)]^{0.6}}\right)^{-1}
$$

The quantity $K$ is related to $\widetilde{m}_{1}$ simply by:

$$
K=\left.\frac{1}{2} D\right|_{z=1} \simeq 170 \frac{\widetilde{m}_{1}}{\mathrm{eV}}
$$

The expression (16) is represented in the figure 1 with the triangle line. One can see that it overestimates the efficiency factor by $\sim 7$. This is not surprising because this analytical approximation takes into account only the inverse decays in the wash out term and it neglects the other processes that are equivalently important. A more specific analytical approach was 
described in [13] and the result for $\kappa_{0}\left(\widetilde{m}_{1}\right)$ is represented in the figure 1 with the starry line ${ }^{2}$. One can see that it better agrees with the numerical results but it still overestimates them by a factor $2-3$.

For large values of $M_{1}$ the efficiency factor depends also on $M_{1}$ itself and actually for $\left.M_{1} \gtrsim 10^{14} \mathrm{GeV}(0.1 \mathrm{eV}) / \bar{m}\right)^{2}$ there is a suppression in the regime for large $\widetilde{m}_{1}$. The suppression is due to a term $\Delta W \propto M_{1} \bar{m}^{2} / z^{2}$, originating from the RH neutrinos mediated processes, that, for large $M_{1}$, dominates in the total wash-out term $W$ [1]. This term suppresses exponentially the baryon asymmetry yielding a term $\exp \left(-\operatorname{const} M_{1} \bar{m}^{2} / \bar{z}\right)$ in the efficiency factor, where $\bar{z}$ is that value of $z$, larger than 1 , at which $\Delta W$ starts to dominate and it can depend only on $M_{1}, \bar{m}$ and $\widetilde{m}_{1}$. Thus, in the most general case, the final baryon asymmetry can be described in terms of only four parameters: $\varepsilon_{1}, \widetilde{m}_{1}, M_{1}$ and $\bar{m}$.

\section{THE SURFACE OF MAXIMUM BARYON ASYMMETRY}

In order to obtain a prediction for $\eta_{B 0}$, to be compared with the measured value $\eta_{B 0}^{C M B}$ in the Eq.(3), one has to multiply $N_{B-L}^{\text {fin }}$ for the fraction of the $B-L$ asymmetry that is converted into baryons by the sphaleron processes, given by a factor $28 / 79 \simeq 1 / 3$, and divide for the dilution factor $f=N_{\gamma}^{\star} / N_{\gamma}^{0}$. This takes into account that the generated baryon asymmetry gets diluted compared to the number of photons that are produced in the annihilations of all standard model particle species. If one assumes a standard thermal history of the early Universe then $f \simeq 28$ and in the end one gets the simple relation

$$
\eta_{B 0} \simeq-10^{-2} \varepsilon_{1} \kappa_{0}
$$

A first trivial model independent bound on $\eta_{B 0}$ is obtained considering that $\left|\varepsilon_{1}\right| \leq 1$ and thus $\eta_{B 0} \lesssim 10^{-2} \kappa_{0}$. It is however possible to show a more stringent bound on $\left|\varepsilon_{1}\right|[14,13,15,1,2]$ :

$$
\left|\varepsilon_{1}\right|<\frac{3}{16 \pi} \frac{M_{1}}{\mathrm{v}^{2}} \frac{m_{3}^{2}-m_{1}^{2}}{m_{3}}=10^{-6}\left(\frac{M_{1}}{10^{10} \mathrm{GeV}}\right) \beta
$$

From neutrino mixing experiments $m_{3}^{2}-m_{1}^{2}=\Delta m_{\mathrm{atm}}^{2}+\Delta m_{\mathrm{sol}}^{2}$ and one can write:

$$
\beta \simeq \frac{\Delta m_{\mathrm{atm}}^{2}+\Delta m_{\mathrm{sol}}^{2}}{0.051 \mathrm{eVm}}
$$

For example in the case of hierarchical neutrinos, for $m_{1}=0$, one has $m_{3}=\sqrt{\Delta m_{\mathrm{atm}}^{2}+\Delta m_{\mathrm{sol}}^{2}}$ and $\beta \simeq 1$. While in the case of quasi degenerate neutrinos with $\bar{m} \simeq 1 \mathrm{eV}$, one has $m_{3} \simeq 0.58 \mathrm{eV}$

\footnotetext{
${ }^{2}$ We deduced it from the figure 1 in [13] interpolating the points for $M_{1}=10^{8} \mathrm{GeV}$. Strangely the result does not correspond to the analytical expression that is given in the text and numbered as Eq. (4.3).
} 


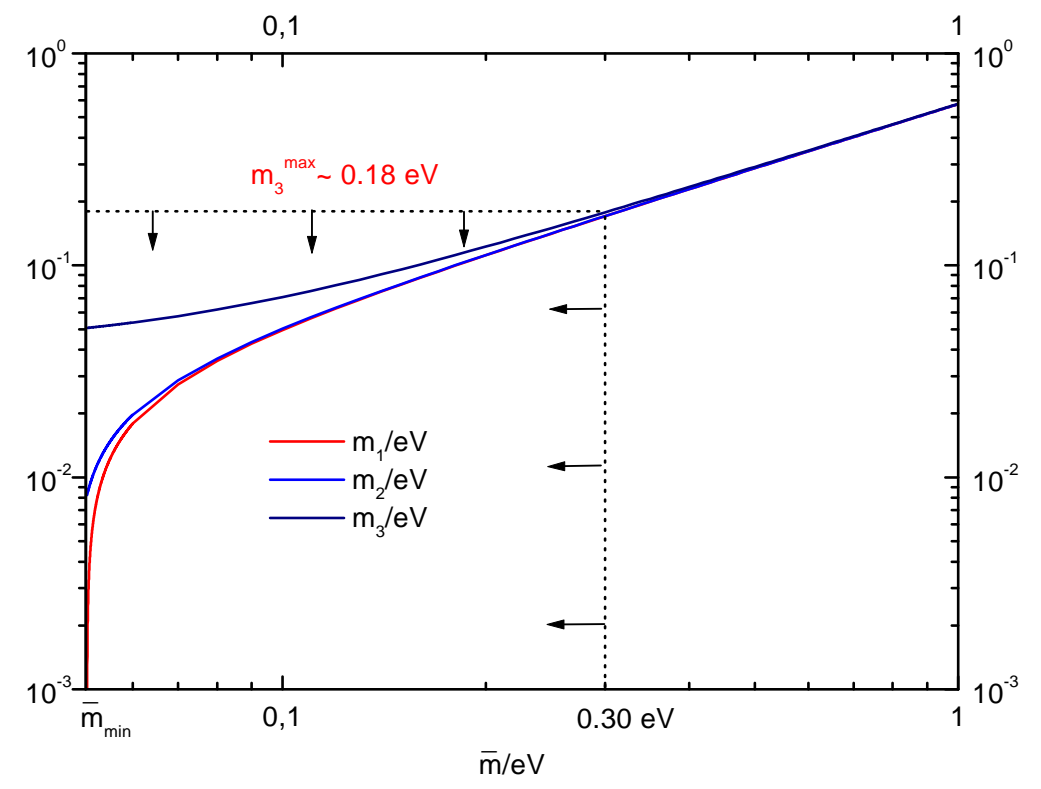

FIGURE 2. Dependence of the light neutrino masses on $\bar{m}$ in the case of normal hierarchy.

and $\beta \simeq 0.1$. In general $m_{3}$, in the Eq. (20), has to be regarded as a function of $\bar{m}$. This is true if one considers the quantities $m_{3}^{2}-m_{2}^{2}$ and $m_{2}^{2}-m_{1}^{2}$ fixed by the solar and atmospheric neutrino experiments. There are two possibilities. In the case of normal hierarchy

$$
\begin{aligned}
& m_{3}^{2}-m_{2}^{2}=\Delta m_{\mathrm{atm}}^{2} \\
& m_{2}^{2}-m_{1}^{2}=\Delta m_{\mathrm{sol}}^{2}
\end{aligned}
$$

and inverting the Eq.'s (8), (21) and (22) one finds:

$$
\begin{aligned}
& m_{3}^{2}=\frac{1}{3}\left(\bar{m}^{2}+2 \Delta m_{\mathrm{atm}}^{2}+\Delta m_{\mathrm{sol}}^{2}\right), \\
& m_{2}^{2}=\frac{1}{3}\left(\bar{m}^{2}-\Delta m_{\mathrm{atm}}^{2}+\Delta m_{\mathrm{sol}}^{2}\right), \\
& m_{1}^{2}=\frac{1}{3}\left(\bar{m}^{2}-\Delta m_{\mathrm{atm}}^{2}-2 \Delta m_{\mathrm{sol}}^{2}\right) .
\end{aligned}
$$

These relations are plotted in figure 2. In the case of inverted hierarchy

$$
\begin{aligned}
m_{3}^{2}-m_{2}^{2} & =\Delta m_{\mathrm{sol}}^{2}, \\
m_{2}^{2}-m_{1}^{2} & =\Delta m_{\mathrm{atm}}^{2}
\end{aligned}
$$

and inverting the Eq.'s (8), (26) and (27) one finds:

$$
m_{3}^{2}=\frac{1}{3}\left(\bar{m}^{2}+\Delta m_{\mathrm{atm}}^{2}+2 \Delta m_{\mathrm{sol}}^{2}\right)
$$




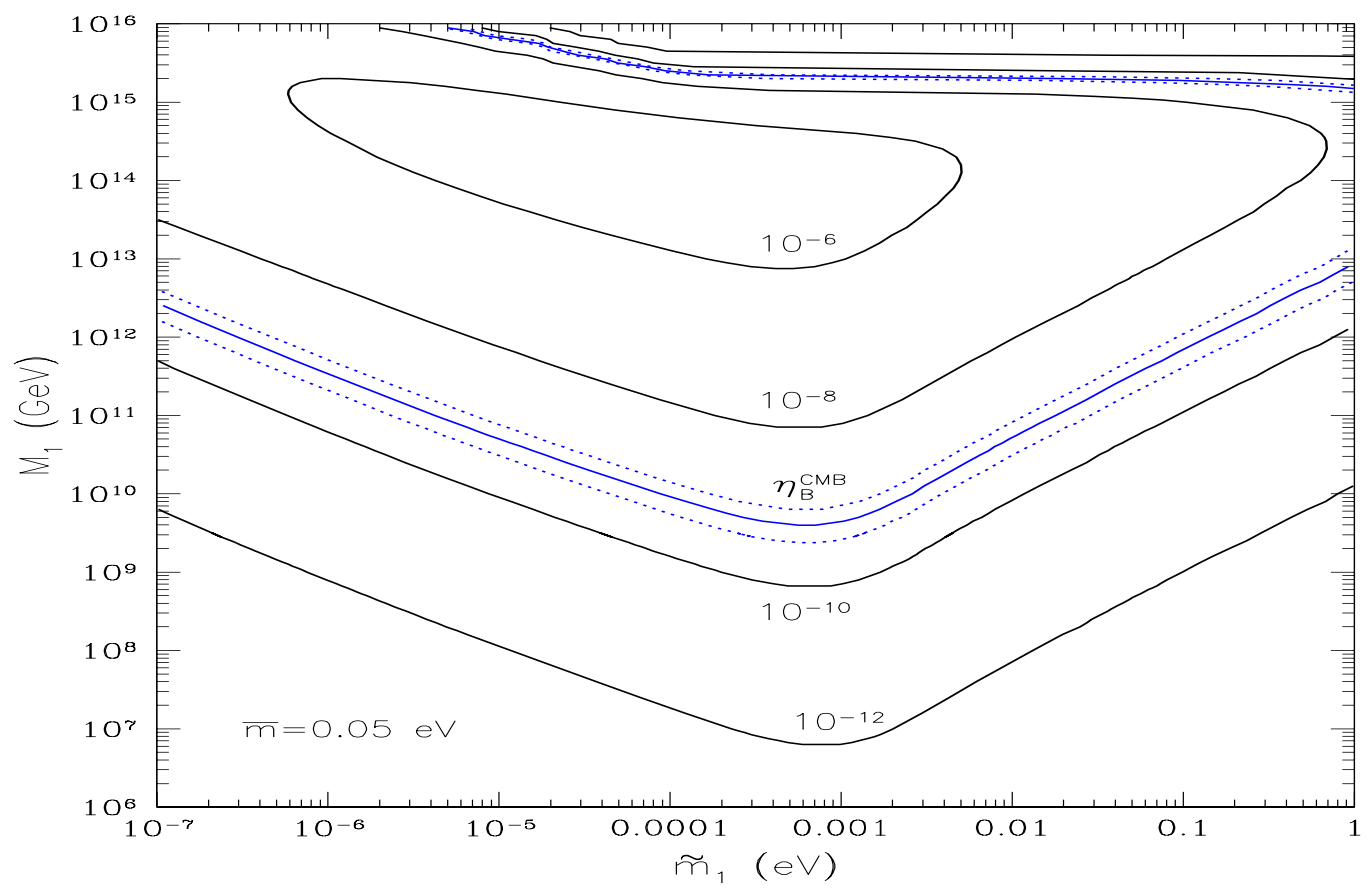

FIGURE 3. Lines of constant $\eta_{B 0}^{\max }$ in the plane $\left(\widetilde{m}_{1}, M_{1}\right)$ (from [1]).

$$
\begin{aligned}
& m_{2}^{2}=\frac{1}{3}\left(\bar{m}^{2}+\Delta m_{\mathrm{atm}}^{2}-\Delta m_{\mathrm{sol}}^{2}\right) \\
& m_{1}^{2}=\frac{1}{3}\left(\bar{m}^{2}-2 \Delta m_{\mathrm{atm}}^{2}-\Delta m_{\mathrm{sol}}^{2}\right) .
\end{aligned}
$$

Using the relation $m_{3}(\bar{m})$ in the Eq.(20) one immediately gets the general dependence of $\beta$ on $\bar{m}$.

From the CP bound, Eq. (19), one can see that, given the atmospheric neutrino mass scale, the mass of the lightest RH neutrino cannot be higher than $10^{16} \mathrm{GeV}$. This because otherwise $\left|\varepsilon_{1}\right|$ would be, absurdly, higher than 1 . This has to be also consistently derived within the seesaw formula. It is certainly true in the oversimplified case of one generation see-saw formula: if $m \gtrsim 0.05 \mathrm{eV}$ then $M \lesssim 10^{15} \mathrm{GeV}$. For three generations the result is analogous and again consistent with the CP bound (see for example [16]) as it has to be. Thus in the end one can express the maximum baryon asymmetry in terms of just three parameters $\widetilde{m}_{1}, M_{1}$ and $\bar{m}$ :

$$
\eta_{B 0}^{\max } \simeq 10^{-8} \beta(\bar{m})\left(\frac{M_{1}}{10^{10} \mathrm{GeV}}\right) \kappa_{0}\left(\widetilde{m}_{1}, M_{1}, \bar{m}\right)
$$

This is the surface of maximum baryon asymmetry and the CMB constraint is given by the requirement that $\eta_{B 0}^{\max } \geq \eta_{B 0}^{C M B}$. In figure 3 we show the iso- $\eta_{B 0}^{\max }$ lines in the plane $\left(\widetilde{m}_{1}, M_{1}\right)$ in the case of a zero initial abundance of $N_{1}$ 's and for $\bar{m}$ equal to its minimum value that is obtained 
in the case of normal hierarchy:

$$
\bar{m}_{\mathrm{min}} \equiv \sqrt{\Delta m_{\mathrm{atm}}^{2}+2 \Delta m_{\mathrm{sol}}^{2}} \simeq 0.05 \mathrm{eV}
$$

This value implies $\beta \simeq 1$ in the bound (19) on $\left|\varepsilon_{1}\right|$ and also $m_{1}=0$. The allowed region lies within the contour line for $\eta_{B 0}^{\max }=\left(\eta_{B 0}^{C M B}\right)_{\text {low }}$ (the external dotted line). There is clearly a lower bound on $M_{1}$. This can be directly obtained from the Eq. (31) imposing $\kappa_{0}$ and $\beta \leq 1$ [1]:

$$
T_{L} \simeq M_{1} \geq 10^{8} \mathrm{GeV} \frac{\eta_{B 0}^{C M B}}{10^{-10}} \gtrsim 4 \times 10^{8} \mathrm{GeV}
$$

at $\sim 2 \sigma$ from the Eq. (3). In the case of a zero initial abundance one can see from the figure 1 that $\kappa_{0} \leq 0.16$ and thus a more stringent constraint, as visible in figure 3 , follows:

$$
T_{L} \simeq M_{1} \geq 6.25 \times 10^{8} \mathrm{GeV} \frac{\eta_{B 0}^{C M B}}{10^{-10}} \gtrsim 2.5 \times 10^{9} \mathrm{GeV}
$$

\section{BOUND ON NEUTRINO MASSES}

If $\bar{m}$ increases then the allowed region shrinks. This happens both because the bound on the CP asymmetry $\left|\varepsilon_{1}\right|$ gets more restrictive ( $\beta$ gets smaller in the Eq. (19) ) and because the action of the wash-out term $\Delta W \propto M_{1} \bar{m}^{2}$ gets stronger. At the same time if $\bar{m}>\bar{m}_{\min }$ then $m_{1}>0$ and in this case it is possible to show another important constraint [17]:

$$
\widetilde{m}_{1} \geq m_{1}
$$

Increasing $\bar{m}$ there will be a value for which the two constraints together cannot be simultaneously satisfied. In figure 4 it is clearly shown that, with a precision of $0.01 \mathrm{eV}$, this value is given by $\bar{m}=0.30 \mathrm{eV}[2]$ and this can be considered an upper limit on $\bar{m}$ for leptogenesis to explain the observed baryon asymmetry. From figure 2 one can see that in terms of light neutrino masses this limit corresponds to have $m_{3} \lesssim 0.18 \mathrm{eV}$ and $m_{1} \simeq m_{2} \lesssim 0.17 \mathrm{eV}$ and this means that leptogenesis is incompatible with quasi-degenerate light neutrinos.

This conclusion has relevant implications for those future experiments that will try to determine the absolute mass scale of neutrinos. There are three classes of such experiments: tritium $\beta$ decay experiments, neutrinoless double $\beta$ decay experiments and cosmological experiments.

The tritium $\beta$ decay experiments are sensitive to an effective electron neutrino mass given by:

$$
m_{v_{e}}=\sqrt{\sum_{i=1}^{3}\left|U_{e i}\right|^{2} m_{i}^{2}}
$$

where the $U_{e i}$ are the elements of the mixing matrix for the electron neutrino flavour. Since $U$ is unitary then $m_{v_{e}} \leq m_{3}$. Therefore the leptogenesis bound predicts that $m_{v_{e}}<0.18 \mathrm{eV}$. 


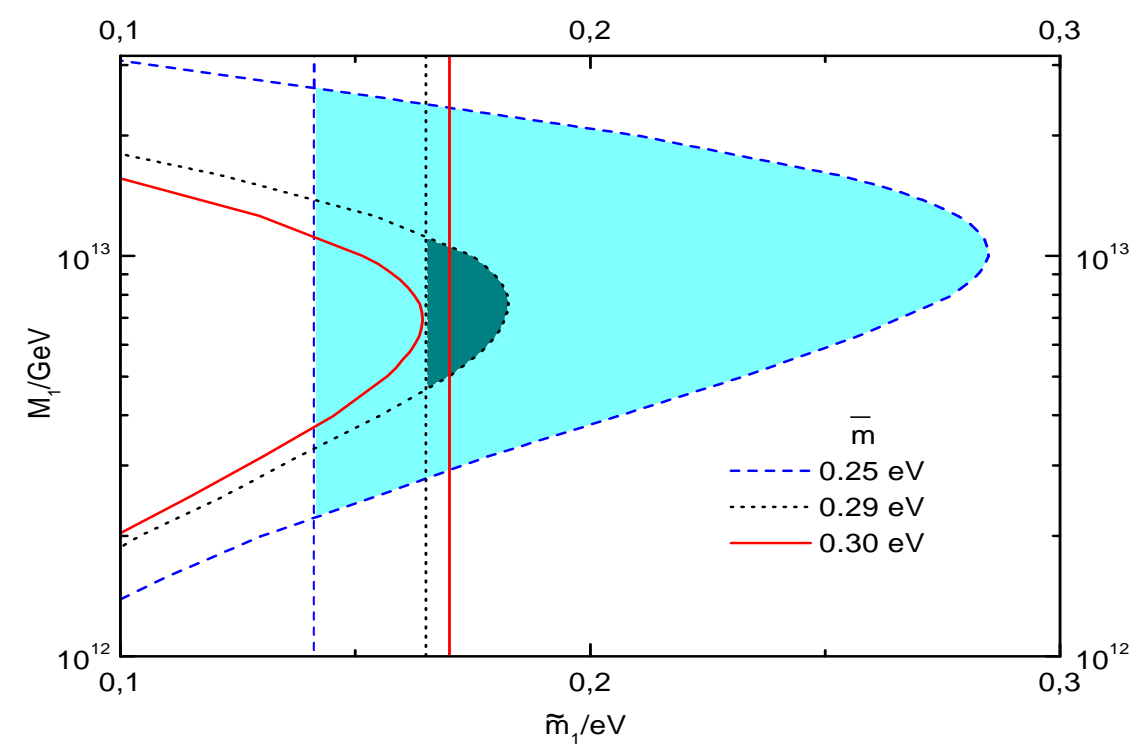

FIGURE 4. Bound on $\bar{m}$ (from [2]). The vertical lines correspond to $\widetilde{m}_{1}=m_{1}$. The other curves are the lines corresponding to $\eta_{B 0}^{\max }=\left(\eta_{B 0}^{C M B}\right)_{\text {low }}$. There is no allowed region (the filled areas) for $\bar{m}=0.30 \mathrm{eV}$.

The KATRIN experiment [18] will be sensitive to $m_{v_{e}} \gtrsim 0.30 \mathrm{eV}$ (at $90 \%$ c.l.) and thus a compatibility with the leptogenesis bound implies no detection of a positive signal.

The cosmological experiments are able to place stringent limits on the sum of neutrino masses. In this case the leptogenesis bound predicts:

$$
\sum_{i} m_{i}<0.52 \mathrm{eV}
$$

The data from the MAP and Planck satellites combined with those from the SLOAN Digital Sky Survey will be sensitive (at $1 \sigma$ ) to $\sum_{i} m_{i} \gtrsim 0.23 \mathrm{eV}$ and $\sum_{i} m_{i} \gtrsim 0.06 \mathrm{eV}$ respectively [19]. Therefore there is certainly room for a positive signal compatible with the leptogenesis bound.

The neutrinoless double $\beta$ decay experiments are sensitive, in the case of Majorana neutrino masses as from the see-saw, to the quantity:

$$
m_{e e}=\left|\sum_{i=1}^{3} U_{e i}^{2} m_{i}\right|
$$

In this case the leptogenesis bound implies:

$$
m_{e e}<0.18 \mathrm{eV}
$$

Future experiments, like the GENIUS project [20], should be sensitive to $m_{e e}>\mathscr{O}(0.01) \mathrm{eV}$ and thus can find a positive signal compatible with the leptogenesis bound. Besides these three 
classes of experiments it is also worthwhile to mention that in the fortunate case that future generations of neutrino telescopes will detect the intense neutrino fluxes needed for the $\mathrm{Z}$ burst scenario to explain the UHECR anomaly and if this anomaly will be confirmed, then we will have another powerful method to measure the absolute scale of neutrino masses, more specifically the highest mass eigenvalue $m_{3}$ [21].

\section{CONCLUSIONS}

It is remarkable that the leptogenesis predictions of the final baryon asymmetry can be expressed in terms of just 4 parameters, in a model independent way. This result relies on two main assumptions: the existence of a mild hierarchy in the masses of the RH neutrinos

$\left(M_{2,3} \gtrsim(2-3) M_{1}\right)$ and that the initial temperature can be assumed to be larger than $M_{1}$. With this parameterization one can easily describe the requirements for a succesfull leptogenesis. A quite precise temperature for leptogenesis seems to emerge to explain the observed baryon asymmetry. The most striking result is that the light neutrino masses cannot be too larger than the atmospheric neutrino mass scale $\sim 0.05 \mathrm{eV}$, thus ruling out the class of quasi degenerate neutrino models. This implies a strong, though negative, prediction on the possibility of future experiments to detect a sub-eV neutrino mass scale, unless their sensitivity can be pushed below $\mathscr{O}(0.1 \mathrm{eV})$. If an evidence for an absolute neutrino mass scale violating the leptogenesis bound will be found then it is possible to relax the main assumptions with an enhancement of the CP asymmetry from a degeneracy of the RH neutrino masses [22] or with a non thermal production of RH neutrinos [23]. In this cases however the nice link between the observed baryon asymmetry and neutrino masses would be lost or controlled by some additional adjustable parameter. If the future experimental results will agree with the leptogenesis bound then the picture will be certainly highly strengthened by the tight conspiracy between the observed baryon asymmetry and neutrino masses.

\section{ACKNOWLEDGMENTS}

This work was supported by the EU Fifth Framework network "Supersymmetry and the Early Universe" (HPRN-CT-2000-00152). All the presented results have been obtained in collaboration with W. Buchmüller and M. Plümacher and more details can be found in [1], [2]. I wish to thank J. Pati who stimulated the comparison of the results on the efficiency factor with those in [11] and J. O'Meara and S. Sarkar for clarifications on the baryon asymmetry measurements. I also wish to thank the following people for their interest in leptogenesis and for nice discussions during my year in the theory group of DESY, during SUSY02, the Third Tropical Workshop and COSMO02 and during my visits in Fermilab, University of Delaware, Bartol Research Institute 
and University of Maryland: K. Abazajian, J. Beacom, N.F. Bell, S. Bludman, A. Brandenburg, L. Covi, J. Formaggio, E. Lisi, R. Fleischer, P.H. Frampton, A. De Gouvea, S. Huber, B. Kyae, C.N. Leung, M. Luty, G. McGregor, R. Mohapatra, H.B. Nielsen, J. Nieves, J. Pati, A. Pilaftsis, A. Ringwald, R.R. Volkas, Q. Shafi, Y. Takanishi, Y.Y.Y. Wong.

\section{REFERENCES}

1. W. Buchmuller, P. Di Bari and M. Plumacher, Nucl. Phys. B 643 (2002) 367.

2. W. Buchmuller, P. Di Bari and M. Plumacher, Phys. Lett. B 547 (2002) 128.

3. J. M. O’Meara, D. Tytler, D. Kirkman, N. Suzuki, J. X. Prochaska, D. Lubin and A. M. Wolfe, Astrophys. J. 552 (2001) 718.

4. B.D. Fields and S. Sarkar in: Review of Particle Physics, Phys. Rev. D 66 (2002) 010001.

5. S. Sarkar, private communication.

6. P. de Bernardis et al., Astrophys. J. 564 (2002) 559; C. Pryke et al., Astrophys. J. 568 (2002) 46; R. Stompor et al., Astrophys. J. 561 (2001) L7; J. A. Rubino-Martin et al., arXiv:astro-ph/0205367; J. L. Sievers et al., astro-ph/0205387.

7. A. Benoit [the Archeops Collaboration], astro-ph/0210306.

8. M. Fukugita and T. Yanagida, Phys. Lett. B 174 (1986) 45.

9. M. A. Luty, Phys. Rev. D 45 (1992) 455.

10. M. Plumacher, Z. Phys. C 74 (1997) 549.

11. E. W. Kolb and M. S. Turner, "The Early Universe”, Addison-Wesley, 1990, chapter 6.

12. H. B. Nielsen and Y. Takanishi, Nucl. Phys. B 636 (2002) 305.

13. R. Barbieri, P. Creminelli, A. Strumia and N. Tetradis, Nucl. Phys. B 575 (2000) 61.

14. W. Buchmuller and T. Yanagida, Phys. Lett. B 445 (1999) 399; K. Hamaguchi, H. Murayama and T. Yanagida, Phys. Rev. D 65 (2002) 043512.

15. S. Davidson and A. Ibarra, Phys. Lett. B 535 (2002) 25.

16. F. Maltoni, J. M. Niczyporuk and S. Willenbrock, Phys. Rev. Lett. 86 (2001) 212.

17. M. Fujii, K. Hamaguchi and T. Yanagida, Phys. Rev. D 65 (2002) 115012.

18. A. Osipowicz et al. [KATRIN Collaboration], arXiv:hep-ex/0109033.

19. S. Hannestad, arXiv:astro-ph/0211106.

20. H. V. Klapdor-Kleingrothaus, Nucl. Phys. Proc. Suppl. 100 (2001) 350.

21. See Z. Fodor, S. D. Katz and A. Ringwald, JHEP 0206 (2002) 046 and references therein.

22. See A. Pilaftsis, Int. J. Mod. Phys. A 14 (1999) 1811 and references therein.

23. G. Lazarides and Q. Shafi, Phys. Lett. B 258 (1991) 305; K. Kumekawa, T. Moroi and T. Yanagida, Prog. Theor. Phys. 92 (1994) 437; G. F. Giudice, M. Peloso, A. Riotto and I. Tkachev, JHEP 9908 (1999) 014. 